\title{
Prediction of Hourly Solar Radiation in Amman-Jordan by Using Artificial Neural Networks
}

\author{
M. A. Hamdan ${ }^{a^{*}}$, E. Abdelhafez ${ }^{\mathrm{b}}$, and O. Ghnaimat ${ }^{c}$ \\ ${ }^{a}$ University of Jordan, Amman, Jordan \\ ${ }^{b}$ Al-Zaytoonah University of Jordan, Amman, Jordan \\ ${ }^{c}$ The Royal Scientific Society, Amman, Jordan
}

\begin{abstract}
In this study, three Artificial Neural Network (ANN) models (Feedforward network, Elman, and Nonlinear Autoregressive Exogenous (NARX)) were used to predict hourly solar radiation in Amman, Jordan. The three models were constructed and tested by using MATLAB software. Meteorological data for the years from 2000 to 2010 were used to train the ANN while the yearly data of 2011 was used to test it. It was found that ANN technique may be used to estimate the hourly solar radiation with an excellent accuracy, and the coefficient of determination of Elman, feedforward and NARX models were found to be $0.97353,0.97376$, and 0.99017 , respectively. The obtained results showed that NARX model has the best ability to predict the required solar data, while Elman and feedforward models have the lowest ability to predict it.
\end{abstract}

Keywords: Solar Radiation, Artificial Neural Networks, Prediction

\section{Introduction}

Due to increasing costs of fossil fuels, uncertainty of availability, increasing environmental pollution, the green sources of energy are being encouraged [1]. Examples of these green sources include wind, hydropower, biomass, geothermal, and most important is solar energy. However, Global Solar Radiation (GSR) data is not readily available and accurate estimation of solar radiation is required for many areas of research in various engineering fields. Further, because of the nature of solar radiation, many parameters can influence both its intensity and its availability and therefore it is difficult to employ analytical methods for its prediction, while multivariate prediction techniques are more suitable [2], among these techniques are; multivariate regression.

Although the concept of ANN analysis had been discovered fifty years ago, it is only in recent decades that application software has been developed to handle practical problems [3]. ANN has many successful applications in various fields of engineering, medicine, economics and many others. Several studies performed ANN modeling for the prediction of solar radiation. Ozgoren et al. [4] used ANN model based on multi-nonlinear regression (MNLR) method to estimate the monthly mean daily sum GSR at any place in Turkey. The results obtained by the ANN model were compared with the actual data, and the mean absolute percentage error (MAPE) was found to be $5.34 \%$ and correlation coefficient $(\mathrm{R})$ value was obtained to be about 0.9936 for the testing data set.

Koca et al. [5] used ANN model estimate the solar radiation parameters for seven cities from Mediterranean region of Anatolia in Turkey. The obtained results indicated that the method could be used by researchers or scientists to design high efficiency solar devices. It was found that number of input parameters was the most effective parameter on estimation of future data on solar radiation.

Rahimikhoob [6] used ANN for estimating the global solar radiation (GSR) as a function of air temperature in a semi-arid environment at the Khuzestan plain, southwest of Iran. The data used in the network training were obtained from a historical series (1994-2001) of daily climatic data. The obtained data was compared with Hargreaaves and Samani (HS) method and it was found ANN technique gave better estimates.

Moghaddamnia et al. [7] described an approach to estimate daily solar radiation at the Brue catchment, United Kingdom. The

${ }^{*}$ Corresponding author. Tel.: +962777498980

E-mail: mhamdan@ju.edu.jo

(C) 2017 International Association for Sharing Knowledge and Sustainability

DOI: $10.5383 /$ ijtee.14.02.003 
nonlinear models used included Local Linear Regression (LLR), multi-layer perceptron, Elman neural network, neural network auto-regressive (NNARX) model with exogenous inputs and adaptive neuro-fuzzy inference system (ANFIS). They concluded that their work was only feasible within the time and resources constrain, due to the Gama Test role in reducing the huge workload of the trial and error processes. They also concluded that the ANFIS model did not have the ability for precise solar radiation estimation, while the LLR and NNARX models are the most suitable models for this study area.

Rehman and Mohandes [1] also used ANN specifically the feedforward type using backpropagation algorithm for estimating GSR as a function of air temperature and relative humidity at Abha city in Saudi Arabia. The estimations of GSR were made using three combinations of data sets namely: (i) day of the year and daily maximum air temperature as inputs and GSR as output, (ii) day of the year and daily mean air temperature as inputs and GSR as output and (iii) day of the year, daily mean air temperature and relative humidity as inputs and GSR as output. Obtained results showed that neural networks were well capable of estimating GSR from temperature and relative humidity. Also, it was found that using the relative humidity along with daily mean temperature had the least absolute mean percentage error of $4.49 \%$ compared with $11.8 \%$ when only day of the year and mean temperature were used and $10.3 \%$ when the maximum temperature was used instead of mean temperature.

Hocaoglu et al. [8] referred to ANN as well as linear models to test their novel two- dimensional approach for hourly solar radiation forecasting. The hourly solar radiation data was measured at a metrological station in Turkey within the period from August 2005 to July 2006. It was observed that the two dimensional model had pronounced advantages over the one dimensional representation for both linear and ANN prediction methods as well as that the ANN models were found to achieve better forecasting results than linear prediction filters in both one dimensional and two dimensional.

The main objective of this study is to investigate the ability of ANN to predict the hourly solar radiation data in Amman, Jordan. The models to be used include nonlinear autoregressive exogenous model (NARX), Elman Network and Multilayer feedforward network.

The recorded hourly solar radiation for ten years from 2000 to 2010 were used as training data while the data recorded in the year 2011 was used as testing data. The input hourly solar data was provided by the National Center for Research and development $\backslash$ Energy Research Program, Amman, Jordan.

\section{Artificial Neural Networks (ANN)}

ANNs are computational models, which replicate the function of a biological network, composed of neurons and are used to solve complex functions in various applications [3]. In general, they are composed of three layers, which are an input layer, some hidden layers and an output layer [9]. The advantages of the ANNs are speed, simplicity and ability to train past data to provide the necessary predictions. ANN has been used in a wide range of applications. These include recognition, optimization, clustering, regression and prediction.

To develop an ANN model, there is three steps must be followed. Firstly, the input is introduced with the desired output to the network together. Secondly, the network is trained to estimate the output in the training step finally, the testing step where an estimation of the output data are obtained by using the input data, which are not used in the training step. More details about these steps are found in [9].

In this study, three types of models (feedforward network, Elman, and NARX) were constructed and tested by using MATLAB Software for predicting hourly solar radiation. A brief introduction to these three neural network models to be used in this study is found in (Moghaddamnia et al., 2009). The performance of all models have been carried out using three global statistics: coefficient of determination $\left(\mathrm{R}^{2}\right)$, root mean squared error (RMSE) and mean bias error (MBE).

The RMSE is a quadratic scoring rule, which measures the average magnitude of the error [9]. Root mean square of the error (RMSE) is computed by Eqs.1. Where t $j$ is the target value, oj is output value, and $p$ is the pattern [3]. This coefficient is expressed as

$R M S E=\sqrt{\sum_{j}\left(t_{j}-o_{j}\right)^{2} / p}$

Coefficient of determination $\left(\mathrm{R}^{2}\right)$ is a measure used in statistical model analysis to assess how well a model explains and predicts future outcomes, which is given by eqs.2. The higher the value of $\mathrm{R}^{2}$ is the more accurate of model.

$$
R^{2}=1-\sum_{j}\left(t_{j}-o_{j}\right)^{2} / \sum_{j}\left(o_{j}\right)^{2}
$$

Mean bias error (MBE) is a measure of overall bias error or systematic error. The less the value of MBE is the more accurate of model. MBE is given by the following expression.



\section{Artificial Neural Networks (ANN)}

The most important step of any neural network is the training step, when the input is introduced with the desired output to the network; the network attempts to produce the desired output by initially choosing randomly the weights and bias values. Once the training process is completed, these weights become of meaningful information. When a reasonable level of performance is reached, the training stops and the network uses the weights to make decisions. It is to be noted that, the goal of any training algorithm is to minimize the global error such as mean percentage error, root-mean-square (RMS), and $\mathrm{R}^{2}$ [3]

Important parameters in the evaluation of the performance are the magnitude of the gradient of performance, the number of validation checks and the value of $\mu$ (the learning rate). The values of both validation checks and $\mu$ are used to terminate the training. The gradient will become very small as the training reaches a minimum of the performance. The number of validation checks represents the number of successive iterations that the validation performance fails to decrease. It is to be noted 
that, during the training in MATLAB, the progress is constantly updated. In this study, three types of ANN models with neuron numbers $(11,20,1)$ were constructed and tested by MATLAB software. Previously obtained experimental data of 8670 sample were used as the input of ANN network. Among this data, $40 \%$ was used for training, 30\% for validation and 30\% for testing. Levenberg- Marquardt (LM) algorithm function was used in ANN network. The number of the hidden layer was selected as 20 in this study using trial and error technique. Tangent sigmoid function was applied for the hidden layer, and linear transfer function is used in the output layer. Training parameters used in LM algorithm are shown in table 1 with their values.

Table 1: Training parameters

\begin{tabular}{|c|c|}
\hline Epochs between displays & 1 \\
\hline Maximum number of epochs to train & 800 \\
\hline Maximum time to train in seconds & inf \\
\hline Performance goal & 0 \\
\hline Maximum validation failures & 15 \\
\hline Factor to use for memory/ speed Tradeoff & 1 \\
\hline Minimum gradient error & $1 * 10^{-5}$ \\
\hline Initial $\mu$ & $1 * 10^{-3}$ \\
\hline$\mu$ decrease factor & 0.1 \\
\hline$\mu$ increase factor & 10 \\
\hline Maximum $\mu$ & $1 * 10^{11}$ \\
\hline
\end{tabular}

\subsection{Elman Network}

In Elman network, the training was stopped after 14 epochs with LM function. The mean square error (RMSE) of training period was found to be $75.0560 \mathrm{MJ} / \mathrm{m}^{2} /$ hour, and RMSE of validation period was found to be $77.0160 \mathrm{MJ} / \mathrm{m}^{2} /$ hour.

Variation of the gradient error, value of $\mu$ and validation checks at each epoch are shown in Fig.1, as indicated in this figure, the gradient error is 2025.0423 , value of $\mu$ is $1 * 10^{11}$ and the number of validation checks are 3 at 14 epochs.

Scatter plot of training, validation, test and experimental data are shown in Fig.2, as it may be noticed in this figure, it was found that the values of $\mathrm{R}^{2}$ in training period, validation period, and testing period are $0.9739,0.97309$ and 0.97349 respectively.

The performance at each epoch is shown in Fig. 3. The maximum performance was found to be 593.4612 at epoch number 11 .

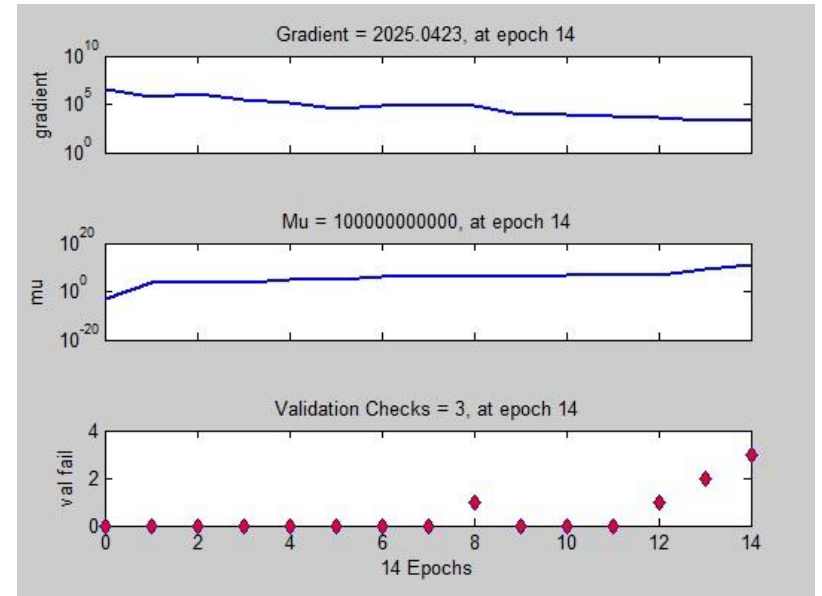

Figure 1: The variation of gradient error and validation checks for Elman Network

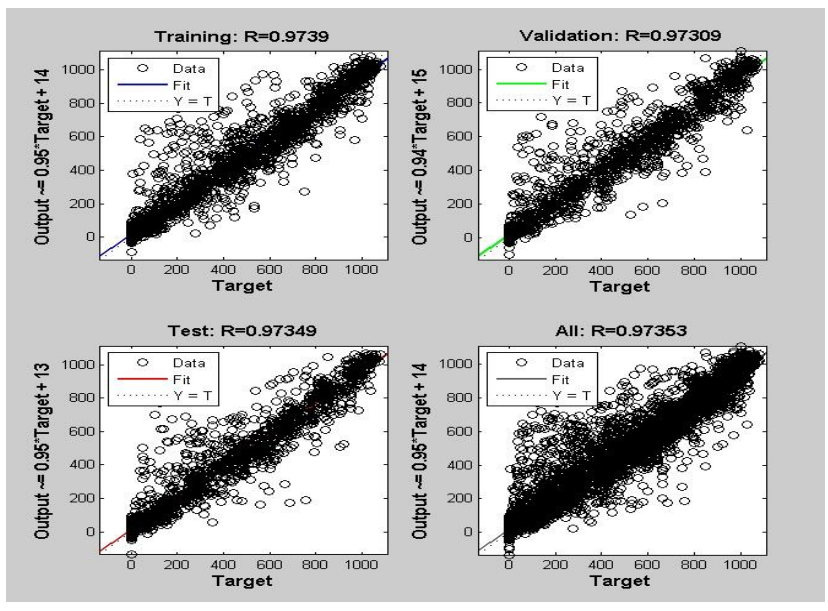

Figure 2: Scatter plot of training, validation, test and all data for Elman Network

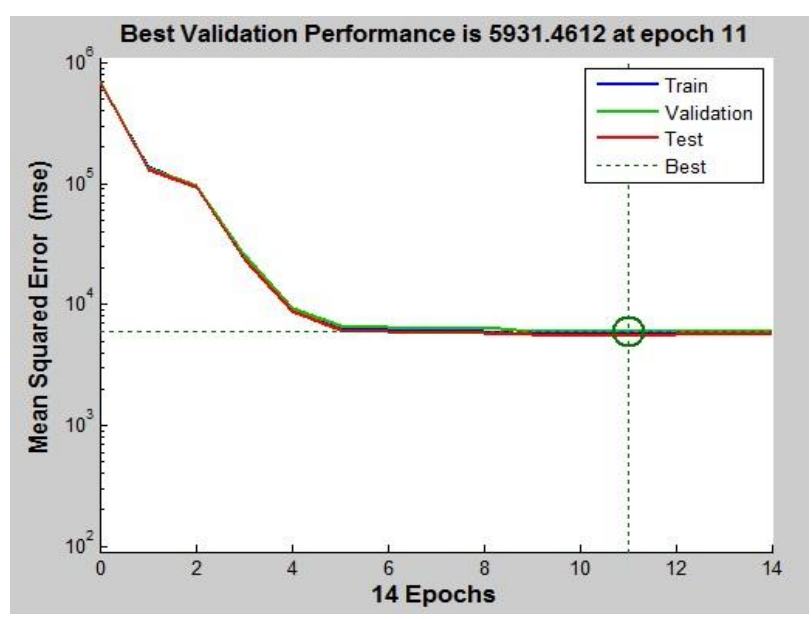

Figure 3: Mean square error at each epoch for Elman Network. 


\subsection{Feedforward Network}

In Feedforward network, the training was stopped after 30 epochs with LM function. The mean square error (RMSE) of training period was found to be $69.0685 \mathrm{MJ} / \mathrm{m}^{2} /$ hour, and RMSE of validation period was found to be $77.9973 \mathrm{MJ} / \mathrm{m}^{2} / \mathrm{hour}$.

Variation of the gradient error, value of $\mu$ and validation checks at each epoch are shown in Fig.4. The gradient error was calculated from this figure to be 13573.9575 , value of $\mu$ is $1^{*} 10^{4}$ and the numbers of validation checks are 15 at 30 epochs.

Scatter plot of training, validation, test and experimental data are shown in Fig.5. As may be noticed in this figure, it was found that the values of $\mathrm{R}^{2}$ in training period, validation period, and testing period are $0.97802,0.97187$ and 0.96999 respectively.

The performance at each epoch is shown in Fig. 6. The maximum performance was found to be 6083.5725 at epoch number 15. Comparing these results with that of Elman network model, it may be noticed that this model gave almost same results as that of Elman model.

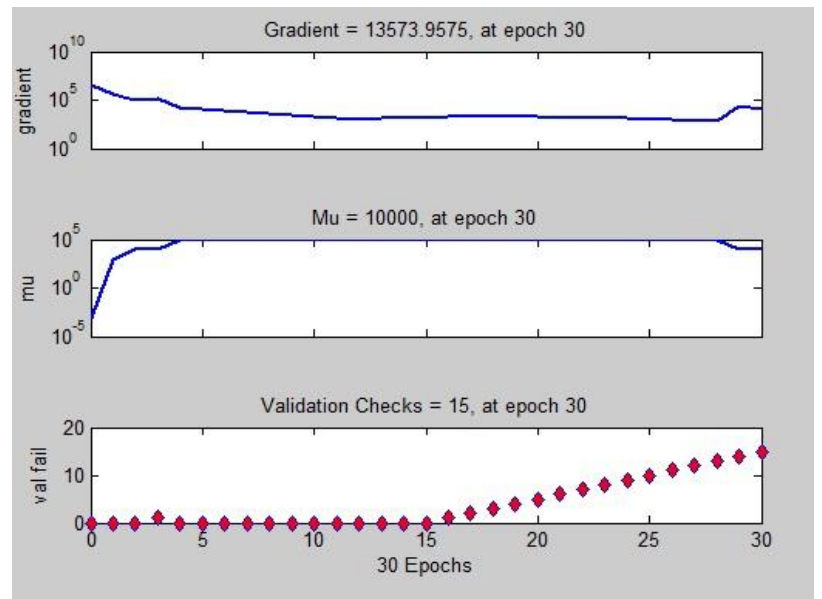

Figure 4: The variation of gradient error and validation checks for Feedforward network.


Figure (5): scatter plot of training, validation, test and all data for Feedforward network.



Figure 6: Mean square error at each epoch for Feedforward network.

\subsection{NARX Network}

In NARX network, the training was stopped after 32 epochs with LM function. The mean square error (RMSE) of training period was found to be $42.8367 \mathrm{MJ} / \mathrm{m} 2 /$ hour, and RMSE of validation period was found to be $48.3991 \mathrm{MJ} / \mathrm{m}^{2} /$ hour.

Variation of the gradient error, value of $\mu$ and validation checks at each epoch are shown in Fig.7. The gradient error is 186.1905 , value of $\mu$ is $1 * 10^{4}$ and the number of validation checks are 15 at 32 epochs.

Scatter plot of training, validation, test and experimental data are shown in Fig.8. As may be notice in this figure, the values of $\mathrm{R}^{2}$ in training period, validation period, and testing period are $0.99157,0.98916$ and 0.98935 , respectively.

The performance at each epoch is shown in Fig. 9. The maximum performance was found to be 2342.4769 at epoch number 17. It may be noticed that this model is characterized by more accurate results compared with those of Elman network and feedforward network. Consequently, this model may be used to predict the data with a high accuracy.

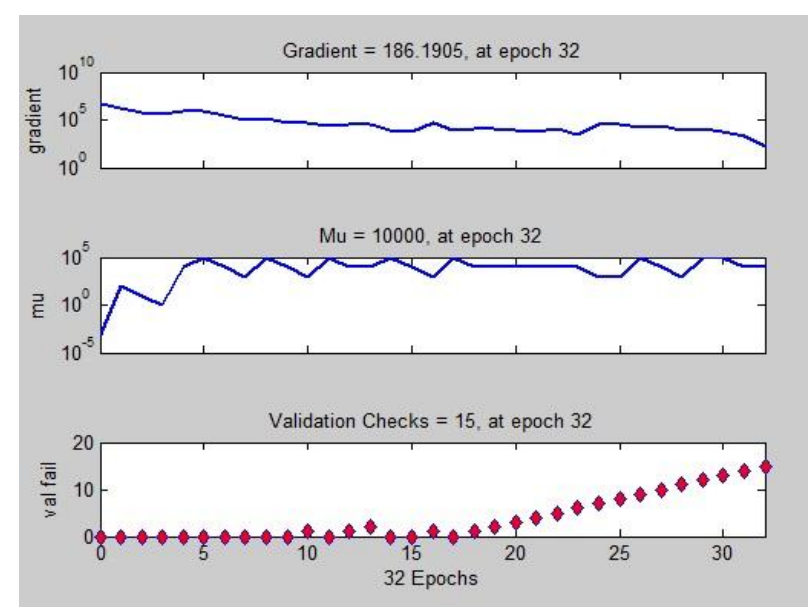

Figure 7: The variation of gradient error and validation checks for NARX network. 

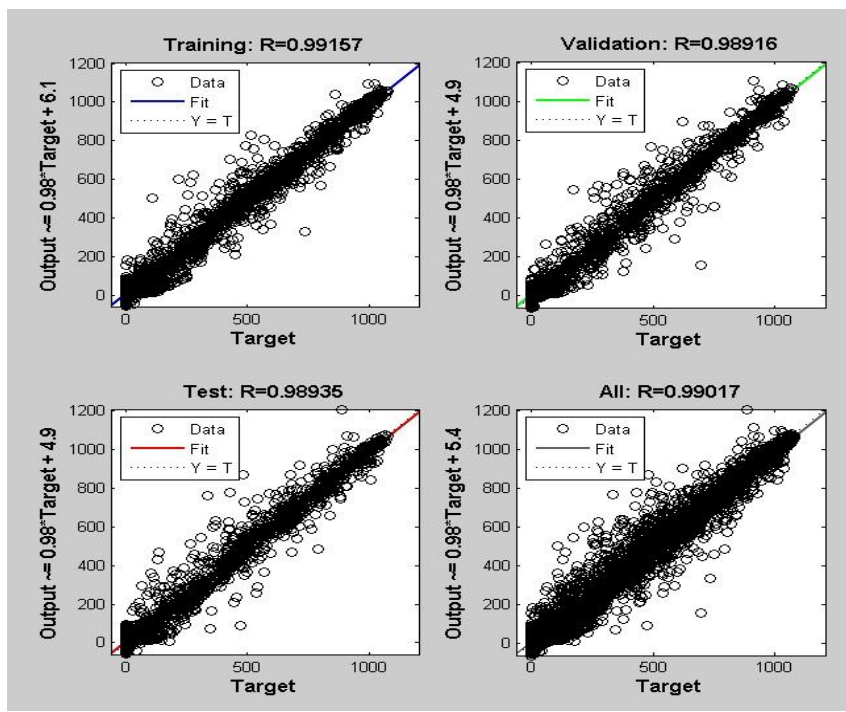

Figure 8: sScatter plot of training, validation, test and all data for NARX network



Figure 9: Mean square error at each epoch for NARX network

The comparison between the obtained experimental data and the estimated one for the three ANN networks for the summer day of $21^{\text {st }}$ of January 2011 and for the winter day of $14^{\text {th }}$ of December 2011 are shown in fig. 10 and 11, respectively. As indicated and in general the prediction in summer day is more efficient than that in the winter, this is due to the effect of cloudy days attenuation to the solar radiation in winter.

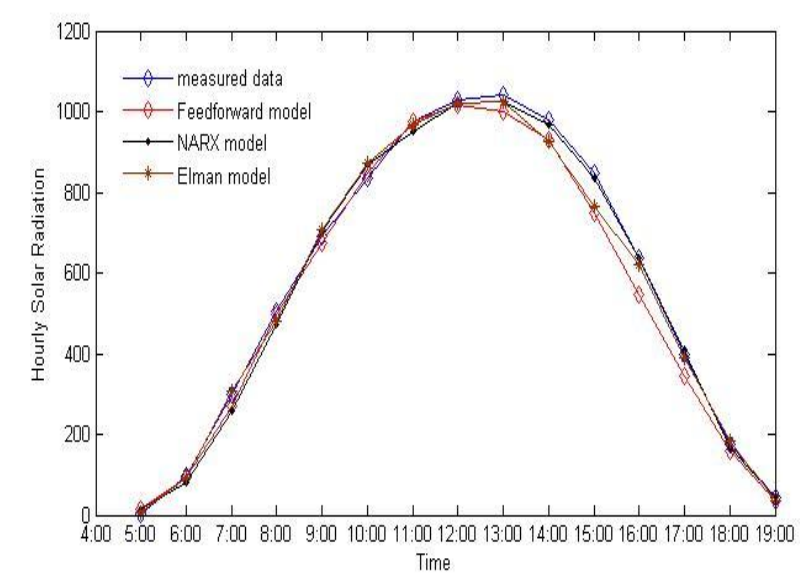

Figure 10: Comparison between experimental and estimated hourly solar radiation at $21 / 6 / 2011$

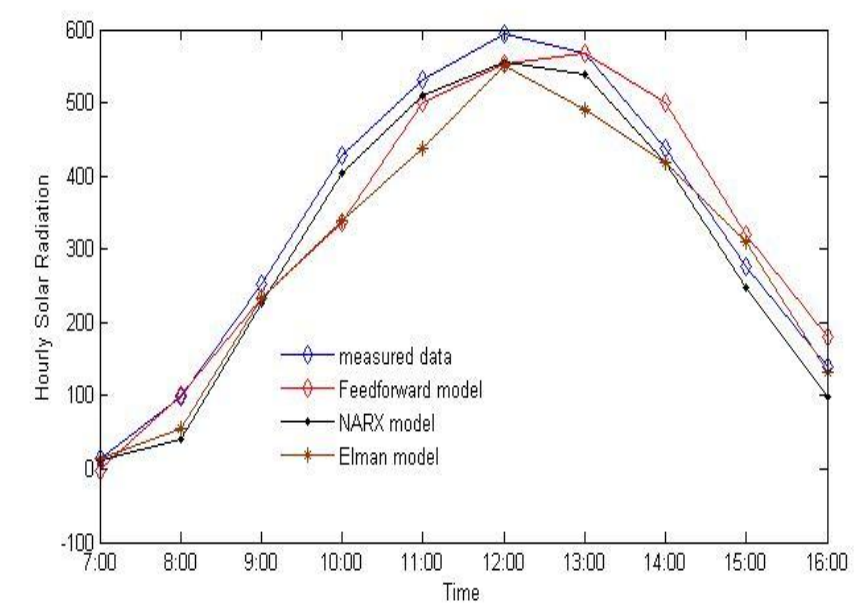

Figure 11: Comparison between experimental and estimated hourly solar radiation at $14 / 12 / 2011$

The three global statistics: coefficient of determination $\left(\mathrm{R}^{2}\right)$, root mean squared error (RMSE) and mean bias error (MBE) are represented in table (2), which may be used to compare the prediction ability of the three models as shown in Table 2 .

Table 2: Comparison of performance of the used models

\begin{tabular}{|c|c|c|c|c|c|c|}
\hline \multirow{2}{*}{ Network Type } & \multicolumn{2}{|c|}{ RMSE } & \multicolumn{2}{c|}{ MBE } & \multicolumn{2}{c|}{$\mathrm{R}^{2}$} \\
\cline { 2 - 7 } & Training & Validation & Training & Validation & Training & Validation \\
\hline Elman & 75.0560 & 77.0160 & 38.0520 & 39.1402 & 0.9739 & 0.97309 \\
\hline Feedforward & 69.0685 & 77.9973 & 33.8347 & 36.5986 & 0.97802 & 0.97187 \\
\hline NARX & 42.8367 & 48.3991 & 25.5612 & 28.5317 & 0.99157 & 0.98916 \\
\hline
\end{tabular}


From the above table and figures for the three ANN model, it may be noticed that NARX model is the most efficient model in the prediction process compared with those of Elman and Feedforward networks. Consequently, this model may be used to estimate the data with a high accuracy.

\section{Conclusion}

In this study, artificial neural network was used to predict hourly solar radiation in Amman, Jordan using hourly solar radiation data that covers the years from 2000 to 2011. ANN technique was used with three different models (feedforward network, Elman, and NARX). These models were constructed and tested using MATLAB software. The obtained results indicate that Elman model has the least ability to predict the hourly solar radiation data. While NARX has the best performance in both training and validation period.

\section{References}

[1] Rehman, S., \& Mohandes, M., (2008). Artificial neural network estimation of global solar radiation using air temperature and relative humidity, Energy Policy, 36, $571-576$.

[2] Kalogirou, S., Michaelides, S., \& Tymvios, F., (2002). Prediction of maximum solar radiation using artificial neural networks, World Renewable Congress VII (WREC2002), Germany.

[3] Sozen, A., Menlik, T., \& Unvar, S., (2008). Determination of efficiency of flat-plate solar collectors using neural network approach, Expert Systems with Applications, 35, 1533-1539.

[4] Ozgoren, M., Bilgili, M., Sahin, B., (2012). Estimation of global solar radiation using ANN over Turkey, Expert Systems with Applications, 39, 5043-5051.

[5] Koca, A., Oztop, H. F., Varol, Y., Koca, G. O., (2011). Estimation of solar radiation using artificial neural networks with different input parameters for Mediterranean region of Anatolia in Turkey, 38, 8756-8762.

[6] Rahimikhoob, A. , (2010). Estimating global solar radiation using artificial neural network and air temperature data in a semi-arid environment, Renewable Energy, 35, 2131-2135.

[7] Moghaddamnia, A. , Remesan, R., Hassanpour Kashani, M., Mohammadi, M., Han, D., \& Piri,J.,( 2009). Comparison of LLR, MLP,Elman, NNARX and ANFIS Models - with a case study in solar radiation estimation, Journal of Atmospheric and Solar-Terrestrial Physics, 71,975-982.

[8] Hocaoglu, F. O., Gerek, O. N., Kurban, M., (2008). Hourly solar radiation forecasting using optimal coefficient 2-D linear filters and feed-forward neural networks, Solar Energy, 82, pp. 714-726.

[9] Caner, M., Gedik, E., and Keçebas, A., (2011). Investigation on thermal performance calculation of two type solar air collectors using artificial neural network, Expert Systems with Applications, 38, $1668-1674$ 